\title{
Lung cancer: how to face the revolution?
}

\author{
Jean-François Morère • Frédérique Penault-Llorca
}

Received: 21 January 2013 / Accepted: 23 January 2013 / Published online: 28 February 2013

(C) Springer-Verlag France 2013

In 2013, the treatment of non-small cell lung cancer finds itself at crossroads. In only a few years, its standard treatment has changed profoundly, moving from a classic chemotherapy with doublets associating a platinum salt and another molecule of third-generation chemotherapy, delivered by intravenous injection, to oral targeted agents for a growing number of patients. This paradigm switch to more "customized" treatment has recently gained momentum.

This personalization of treatment was initially introduced on the basis of clinical and histopathological criteria, and the standard of care was first guided by performance status and age. The treatment of the elderly benefited remarkably from a precise geriatric evaluation and specific therapeutic options in accordance with Balducci's classification system. More recently, the concept of chemotherapy guided by histopathological features was introduced, the efficacy of certain chemotherapeutic agents being restricted to certain specific histologic subtypes of lung tumors.

A better knowledge of the oncogenesis of bronchial carcinomas, together with major improvements in molecular biology tools, has led to a further step in the personalization of treatment. Today, we can routinely identify certain driver molecular abnormalities in tumors, from paraffin blocks. These drivers are essential in the preservation of tumor phenotypes and act as therapeutic targets.

This biological profiling of tumors, combined with their histopathological characterization, has become one of the most essential criteria in focusing the treatment applied to

\footnotetext{
J.-F. Morère $(\square)$

Oncology Department, Paul Brousse Hospital, Villejuif, France

e-mail: jean-francois.morere@pbr.aphp.fr

F. Penault-Llorca

Jean Perrin Center, Clermont-Ferrand, France
}

these bronchial cancers. Pathologists will play a key role in the identification of the molecular targets. Their duties have evolved, and we can observe massive advances in the field of molecular pathology. The major limitation remaining is the quantity of tissue available for the characterization. In response, technology is evolving very rapidly, allowing biomarker multiplexing and the analysis of circulating DNA.

New oncogenic addictions are being identified on a regular basis: initially in adenocarcinoma in nonsmokers, then in adenocarcinoma in former smokers, and finally and more recently, in squamous cell carcinomas.

Fortunately, at the same time, new molecules are focusing on these new targets within the framework of therapeutic trials, confirming in medical practice the new paradigm of the co-development of targeted therapies along with companion tests.

Some of these targetable pathways, e.g., EGFR and EML4-ALK, can already routinely be detected and treated. Others, such as BRAF, ROS1, and KRAS, are next on the list, while yet others are being studied and may emerge to expand the arsenal of lung cancer treatment.

Unfortunately, after a phase of responsiveness to targeted therapy, almost all patients exhibit resistance. In the near future, a better appraisal of the mechanisms underlying resistance to targeted therapies in lung tumors will allow us to develop new agents aiming to counter acquired resistance, delivering better efficacy in patient management.

In this ongoing battle between oncologists and cancer, we now see oncologists proceeding more tactically, working to anticipate the moves of the tumors. The editorial staff of Targeted Oncology thus felt it was important to take time at the beginning of the new year to review these major upheavals (or even revolutions) in the treatment of lung cancer. 
In this issue of Targeted Oncology, D. Planchard will describe the different targets currently screened for in lung tumors, using the example of the French INCalabeled platforms. You will find a meta-analysis of targeted therapies for adenocarcinoma by E. Bria et al. K. Kobayashi presents EGFR targeting for tyrosine kinase inhibitors; R. Pirker explores the use of the same method for monoclonal antibodies. F. Casaluce et al. report on ALK inhibition, while N. Girard discusses the new targets involved. Lastly, Schmid-Bindert et al. give us an update on the use of anti-angiogenics in lung cancer.

Enjoy your issue of Targeted Oncology!

Conflict of interest Frédérique Penault-Llorca declares to be consultant and part of Roche and Pfizer boards. Jean-François Morère declares to be part of Boehringer and Pfizer boards. 